\title{
Challenging Implants Require Tools and Techniques Not Tips and Tricks
}

\author{
Seth J. Worley, MD
}

\section{KEYWORDS}

- Orthodromic snare technique $\bullet$ Antidromic snare technique $\bullet$ Vein selectors $\bullet$ CS atresia

- Anchor balloon • Amplatz wire • Amplatz support wire technique • CS cannulation

\section{KEY POINTS}

- Successful left ventricular (LV) lead implantation requires preparation, tools, and techniques not tips and tricks. The implanting physician must be proactive and not rely on the device company to provide all the necessary tools.

- The Amplatz wire (Cook) + Vertebral Vein Selector is a powerful combination for both initial coronary sinus (CS) cannulation and to implement the support wire technique.

- Patients with CS atresia can be implanted through a persistent vein of Marshall.

- The orthodromic snare technique makes it possible to implant LV leads in small and/or tortuous veins not accessible by other means.

- The antidromic snare technique makes it possible to implant LV leads in target veins where a wire cannot be advanced.

\section{INTRODUCTION}

In some cases, left ventricular (LV) lead implantation can be difficult.

This article focuses on specific issues as they appear in the sequence of the implant. Combining the Cook Amplatz (Medical Bloomington, Indiana, USA) wire with the "Vertebral Vein Selector" is useful both for initial coronary sinus (CS) cannulation (Box 1, Videos 3 and 4) and to stabilize CS access (Box 2, Videos 4, 8, 11, 12, 13, 15). The term "Amplatz Wire-Vertebral Vein Selector technique" refers to the situation whereby the Amplatz wire and the vein selector are used to facilitate CS cannulation, whereas the "Amplatz support wire technique" refers to the situation whereby the wire is used to stabilize CS access (see Box 2).

\section{SET UP}

The trick is to be prepared by having the necessary equipment in the room readily available on a cart (Fig. 1). Box 3 details the equipment on the cart. Proper table position (Fig. 2) and table designed for LV lead implantation (Fig. 3) are also important. ${ }^{1}$ The proper table is also useful when a subclavian occlusion needs crossed and dilated for venous access.

\section{VENOUS ACCESS}

The importance of subclavian venoplasty for venous access is covered in Daniel J. Friedman and Kevin P. Jackson's article, "How to Implant CRT in a Busy Clinical Practice," in this issue of Cardiac Electrophysiology Clinics. ${ }^{2-4}$

Disclosure Statement: Royalties from Merit Medical (South Jordan, Utah, USA) and Pressure Products. Consulting Medtronic (Minneapolis, Minnesota, USA), Abbott, Biotronik.

Cardiac Electrophysiology Division, Medstar Heart and Vascular Institute, 110 Irving Street Northwest, Suite 5A12, Washington, DC 20010, USA

E-mail address: seth@mcworley.com 


\section{Box 1 \\ Amplatz vertebral vein selector coronary sinus cannulation technique step by step}

The Amplatz Vertebral Vein Selector CS cannulation tech wire technique works for all catheters used for CS access.

Advance a "Vertebral Vein Selector" over an angled 0.035-in glide wire deep into the CS. When using the Worley sheath, this will be through the braided core.

Keeping the Vertebral Vein Selector deep in the CS, replace the glide wire with a 0.035 -in 180 $\mathrm{cm} J$ tip Cook Amplatz extra stiff wire. The short taper of the Cook Amplatz is important and not found in J tip Amplatz wires from other companies.

The combination of the Cook Amplatz wire and the Vertebral Vein selector deep in the CS provides a rail over which the sheath can be advanced despite stenosis or tortuosity.

Once the CS is cannulated, the Amplatz wire can be left in place if the 9-French Worley sheath is used for CS access.

\section{LOCATING THE CORONARY SINUS}

Trying to locate the CS with an electrophysiology catheter or wire (poke and pray), although often successful, is intrinsically limited. When you "poke and pray" and do not locate the CS, nothing is learned. Locating the CS via catheter manipulation with contrast injection (described in the video 1) is intrinsically superior but requires a change in approach for many. ${ }^{5}$

Problem = locating the CS for CS Cannulation (Fig. 4); solution = contrast injection and catheter manipulation to use the Eustachian ridge and Thebesian valve to facilitate CS cannulation. Watch Video 1 for the details on CS cannulation using the Worley CSG with braided core (https://www. youtube.com/watch?v=OE0yimc13uQ).

Problem $=$ unable to locate the CS despite using contrast and catheter manipulation: possible CS atresia (Fig. 5); solution = look for a persistent vein of Marshall through which to implant the LV lead. Watch Video 2 for details of LV lead implantation in a patient with CS atresia and a persistent vein of Marshall (https://www.youtube.com/ watch? $\mathrm{v}=1 \mathrm{mLRpw}(1 \mathrm{k} 8 \mathrm{~A})$. It is more common than first thought.

\section{CORONARY SINUS CANNULATION ONCE THE CORONARY SINUS IS IDENTIFIED}

Problem $=$ difficult to advance into the CS (Fig. 6); solution $=$ "Vertebral Vein Selector" combined
Box 2

Amplatz support wire technique step by step

Amplatz wires are stiff and thus do not advance well into the CS but provide excellent support once in place. The Vertebral Vein selector serves as a conduit through which to introduce the Amplatz wire. To avoid perforation, always use a J tip Amplatz wire.

The 9-French internal diameter "Worley" sheath (WOR-CSG-B1-09 Merit Medical) provides the option to use the Amplatz support wire technique. The support wire technique is not an option with 7-French CS access catheters provided by the device companies.

Advance a "Vertebral Vein Selector" over an angled 0.035-in glide wire deep into the CS.

Keeping the Vertebral Vein Selector deep in the CS, replace the glide wire with a 0.035 -in 180$\mathrm{cm} J$ tip Cook Amplatz extra stiff wire. The short taper of the Cook Amplatz is important and not found in J tip Amplatz wires from other companies.

Remove the "Vertebral Vein Selector" keeping the Amplatz wire in place.

With the 9-French sheath stabilized by the Amplatz wire, the Vein Selector telescoped inside the subselector is advanced beside the Amplatz wire. The shape of the vein selector depends on the takeoff of the target vein. For target veins at the ostium of the CS, the sheath is withdrawn to uncover the target vein without loss of access.

with the Cook Amplatz wire. Watch Video 3 for details on how to implement the technique (https:// www.youtube.com/watch?v=SB4nNBmy-3g).

Problem $=$ difficult $C S$ to advance and unstable CS access with huge right atrium (RA) (Fig. 7); solution = Jumbo Worley Sheath and Cook Amplatz support wire. Watch Video 4 to see how (https://www.youtube.com/watch?v=5P7gJltePP8). In many cases just switching from a standard sheath to the Jumbo Worley CSG solves the problem.

Problem = drain pipe CS unable to advance sheath into the CS (Fig. 8); solution = the anchor balloon technique. ${ }^{6}$ Watch video 5 to learn how to implement the anchor balloon technique for CS cannulation (https://www.youtube.com/ watch? $v=h m X t c s V j s y w)$. The anchor balloon can also be used to advance a subselector into a difficult target vein.

Problem $=$ Vieussens valve prevents access to the great cardiac vein (Fig. 9); solution $=$ "Vertebral Vein Selector" to advance past the Vieussens valve. Watch Video 6 for details on 


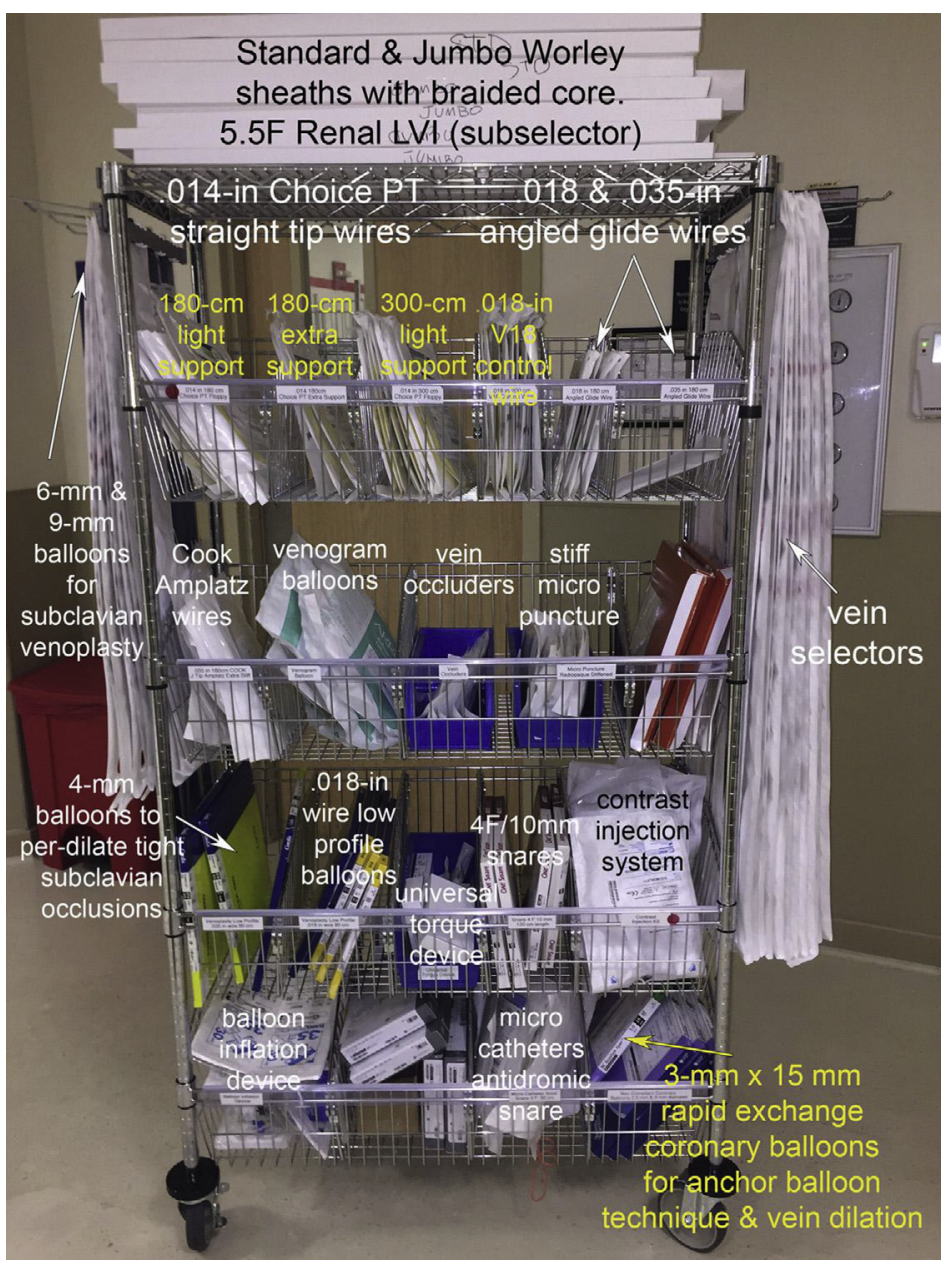

Fig. 1. Cart with the equipment necessary for both basic and challenging LV lead implantation as well as subclavian venoplasty. (See Box 1 for complete details.) (Courtesy of Seth J. Worley, MD, Washington, DC.)

how to use the "Vertebral Vein Selector" to cross the Vieussens valve (https://www.youtube.com/ watch? $v=$ SdTEB3R6W8M\&t=5s).

\section{OCCLUSIVE CORONARY SINUS VENOGRAM}

Start by using full-strength contrast and a control syringe for an adequate CS venogram. Insure the balloon is occlusive by placing the balloon above the Vieussens valve. Do not assume that it is safe to inflate the balloon because it was advanced over a wire; you could be in the vein of Marshall, so always do a gentle test injection first. Watch for retrograde filling of proximal veins.

Problem $=$ high outputs and phrenic pacing throughout the only viable target vein identified on CS venogram (Fig. 10); solution = full-strength contrast injection through a "Vein Selector" advanced over a wire into the branch to demonstrate an alternative target vein off of the CS.
Watch Video 7 for details on selective target vein venography using a Vein Selector (https://www. youtube.com/watch?v=23nhCd2h9Gw).

Problem $=$ high outputs and phrenic pacing throughout the only viable target vein identified on CS venogram (Fig. 11); solution = full-strength contrast injection through a "Vein Selector" advanced over a wire into the target vein demonstrates a side branch off the target vein. Watch Video 8 for details (https://www.youtube.com/ watch?v=mWVeJIYC92s).

\section{LEFT VENTRICULAR LEAD IMPLANTATION}

Problem = using the "poke-and-pray" approach can be difficult to advance a wire into the target vein (Fig. 12); solution $=3$ vein selector shapes designed to be telescoped into the subselector. Vein selectors attached to the contrast injection system are designed to locate the target vein with a puff of contrast as you might locate the right 
1. Worley Advanced Standard Curve; Order no. WOR-CSG-B1-09 Merit Medical.

2. Worley Advanced Jumbo Curve; Order no. WOR-CSG-B2-09. Merit Medical.

3. Catheters for right side CS access and difficult to locate CS are hand shaped to resemble the braided core. 6-French Boston Scientific (Marlborough, Massachusetts, USA) Runway MP2 (Multipurpose 2) Ref. H74938969390 (Alternative no. 1 = 6 French Boston Scientific Mach 1 MP2 Order 34356-39, Alternative no. 2 = 6 French Medtronic (Minneapolis, Minnesota, USA) MB1 Z2 Guiding Catheter Medtronic Vascular Z26MB1. Alternative no. 3 = 6 French Medtronic Launcher MB2 Ref. LA6MB2. Alternative no. 4 = 6 French Cordis (Milpitas, California USA) Vista Bright Tip MPB 1 Ref. 670-275-00).

4. Worley Standard Vein Selector $(5$ French $\times 75 \mathrm{~cm}$ ) Merit Order no. $57538 C S-$ WOR Merit Medical.

5. Worley Vert Vein Selector $(5$ French $\times 75 \mathrm{~cm}$ ) Merit Order no. 57538CSV-WOR Merit Medical.

6. Worley Hook Vein Selector $(5$ French $\times 75 \mathrm{~cm}$ ) Merit Order no. $57538 C S H K-W O R$ Merit Medical.

7. Contrast Injection System Worley Advanced Kit 1 CAK 1 (comes with contrast bowl and labels) (order no. K12-WORLEY1 Merit Medical).

8. Subselector = 5.5-French ID Worley advanced Telescoping LVI, Order no. WORLVI-75-5-62-55-RE.

9. 5-French micropuncture kit with 0.018 Nitinol wire and stiffened radiopaque dilator Merit Medical. Order no. S-MAK501N15BT.

10. 5-French Impress KA 2 Hydrophilic Angiographic Catheter 5-French $65 \mathrm{~cm}$ (Order no. 56538KA2-H Merit Medical) to assist crossing difficult subclavian obstruction.

11. 4-French Impress KA 2 Hydrophilic Angiographic Catheter 4-French 65 cm (Order no. 46538KA2-H Merit Medical).

12. 0.014 -in CholCE PT (Polymer Tip) Straight tip Light Support (Order no. 1211-01 Boston Scientific) (see annotated list in later discussion for options).

13. 0.014-in CholCE PT (Polymer Tip) Straight (not angled) Tip Extra Support (Order no. 12161-01 Boston Scientific).

14. 0.014-in CholCE PT (Polymer Tip) Straight (not angled) tip Light Support $300 \mathrm{~cm}$ Boston Scientific.

15. 0.018 V-18 Control Wire Guidewire with ICE Coating Polymer Tip Hydrophilic (0.018-in 200-cm short taper) Boston Scientific (Catalog no. 46-852).

16. Cook Amplatz Extra Stiff Wire Guide, 0.035-in, 180-cm, 3-mm tip curve Cook (THSCF-35-180-3-AES) (another ref. no. on the package is G03565) (do not substitute).

17. Angled (not straight) polymer tip hydrophilic wire 0.035 in $\times 180 \mathrm{~cm}$ Laureate wire (Order no. LWSTDA35180 Merit Medical) (Glide wire or Terumo).

18. Angled (not straight) polymer tip hydrophilic wire $0.018 \mathrm{in} \times 180 \mathrm{~cm}$ (Laureate Wire Order no. LWSTDA18180 Merit Medical) (Glide wire or Terumo)

19. Snare (Micro) 10-mm loop/4-French snare catheter Merit One Snare ONE 1000 (snare = $10 \mathrm{~mm}$ loop, $120 \mathrm{~cm}$ length; catheter = 4-French $100-\mathrm{cm}$ length inside diameter $1.02 \mathrm{~mm}$ ). This $10-\mathrm{mm}$ snare fits into the 9-French internal diameter CSG Worley beside the vein selector (Order no. ONE100 Merit Medical).

20. Microcatheter for the antidromic technique Merit SureCross Support Catheter (length $90 \mathrm{~cm}$, wire diameter 0.014 in, tip diameter 0.020 in [1.52 French]) Catalog no. SC1490.

21. Subclavian venoplasty balloon $6-\mathrm{mm}$ diameter $\times 4 \mathrm{~cm}$ length $\times 75 \mathrm{~cm}$. CONQUEST Order no. CQ7564 Bard (Covington, Georgia, USA) Peripheral Vascular rated burst pressure 30 atm.

22. Subclavian venoplasty $9 \mathrm{~mm} \times 4 \mathrm{~cm} \times 75 \mathrm{~cm}$ balloon Order no. CQ-7594 Bard Peripheral Vascular rated burst pressure 26 atm.

23. Cordis Powerflex Pro OTW (0.035-in wire) Balloon Catheter: Balloon $4 \mathrm{~mm}$ diameter $\times 40 \mathrm{~mm}$ long; shaft length $80 \mathrm{~cm}$ Order no. 4400404S.

24. Noncompliant rapid exchange coronary balloon, for example, NC Sprinter from Medtronic $3.0 \mathrm{~mm}$ diameter $\times 15$ mm length, Catalog no. H7493912415300 GITN, no. 08714729783374.

25. Cook Needle's Eye Snare alone $=13-\mathrm{mm}$. Femoral Snare and straight 12-French sheath without work station; Ref.: G26518, Ref.: LR-SSN001.

26. Cook Needle's Eye Snare alone $=20-\mathrm{mm}$. Femoral Snare and straight 12-French sheath without work station, Ref.: G26516, Ref: LR-SSN002.

28. 16-French Curved Work Station alone, 16-French Curved Work Station (Femoral Introducer Sheath Set), Ref.: G26566, Ref.: LR-CSS16. 


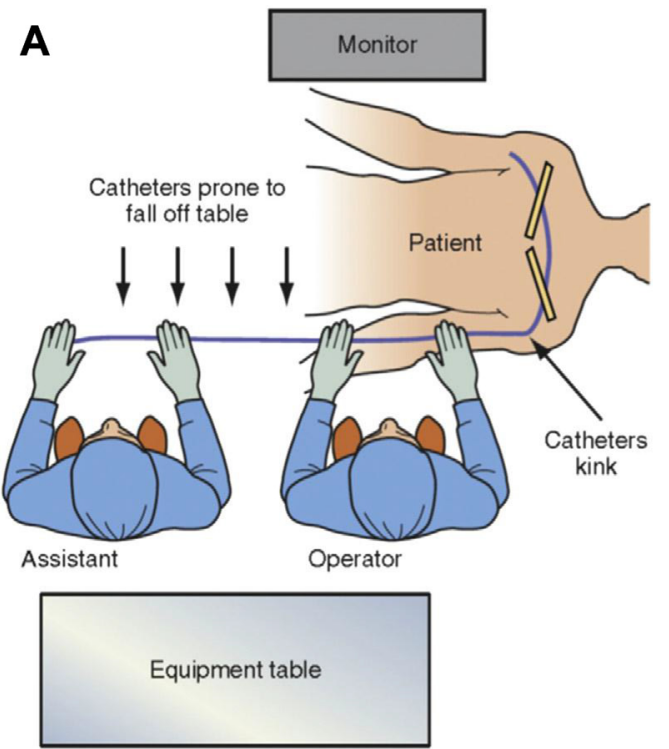

B Catheters don't kink:

fall naturally onto the table

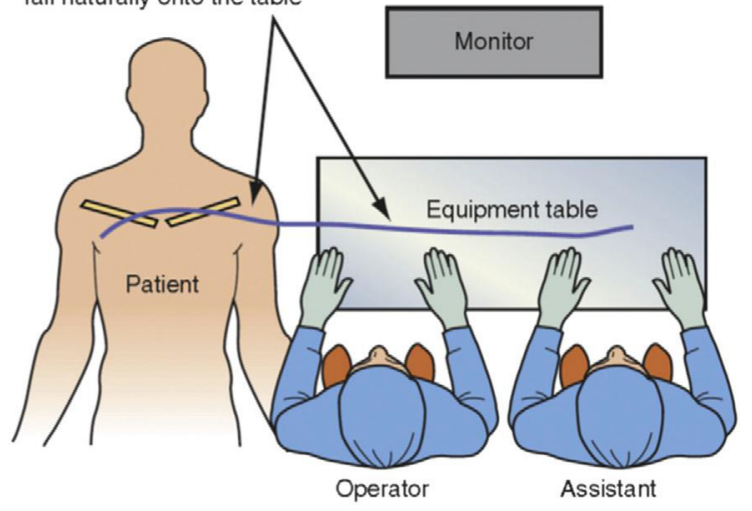

Fig. 2. Importance of table position for successful LV lead implantation. The perpendicular table position improves the ergonomics of catheter and wire exchange; the catheter torque control is improved by removing an acute angle, and wires are less likely to fall off the table. . (Courtesy of Seth J. Worley, MD, Washington, DC.)

coronary artery. Once the vein is located, a wire is advanced. The vein selector is then advanced over the wire deep into the target vein. To facilitate advancing the vein selector into a difficult target vein, up to four 0.014-in angioplasty wires can be advanced through the 0.038-in lumen. The subselector is then advanced over the wire stabilized vein selector deep in the target vein. The vein selector is removed, retaining one wire for LV lead delivery. Watch Video 9 for details on the use of the wire stabilized vein selector (https:// www.youtube.com/watch?v=IsawLqHGq-g).

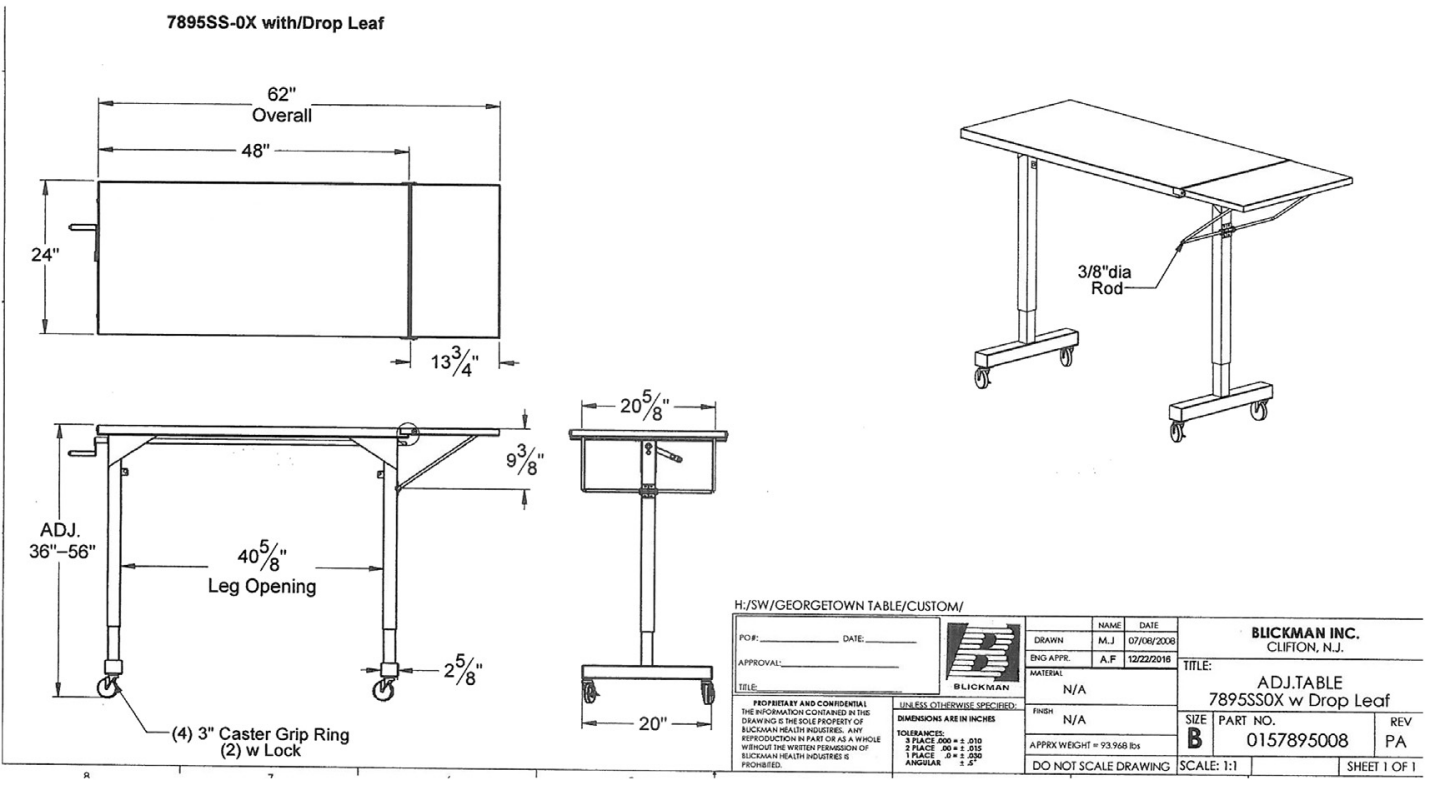

Fig. 3. The table is designed for the interventional approach to device implantation. The table height can be adjusted to the height of the patient table. The extension allows the x-ray tube to be rotated to the right anterior oblique (RAO) without hitting the legs of the table. ADJ, adjustable. (Courtesy of Seth J. Worley, MD, Washington, DC.) 


\section{The tip of the braided core drops into the pocket created by the Eustacian ridge and Thebesian valve}

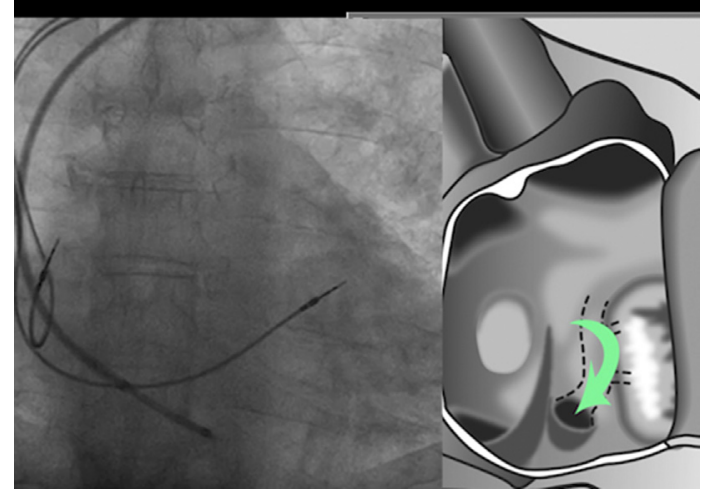

Fig. 4. How to cannulate the CS using catheter manipulation and contrast injection. With counterclokwise torque, the tip of the catheter drops down the annulus into the CS (arrow). The Eustachian ridge and Thebesian valve direct the tip of the catheter into the CS. Watch Video 1 for details (https://www. youtube.com/watch?v=OE0yimc13uQ). (Courtesy of Seth J. Worley, MD, Washington, DC.)

Problem = drain pipe target vein (Fig. 13); solution $=$ the "Hook Vein Selector" telescoped in the renal lateral vein introducer (LVI) subselector plus the use of multiple wires to advance the vein selector deep into the target vein. For details,

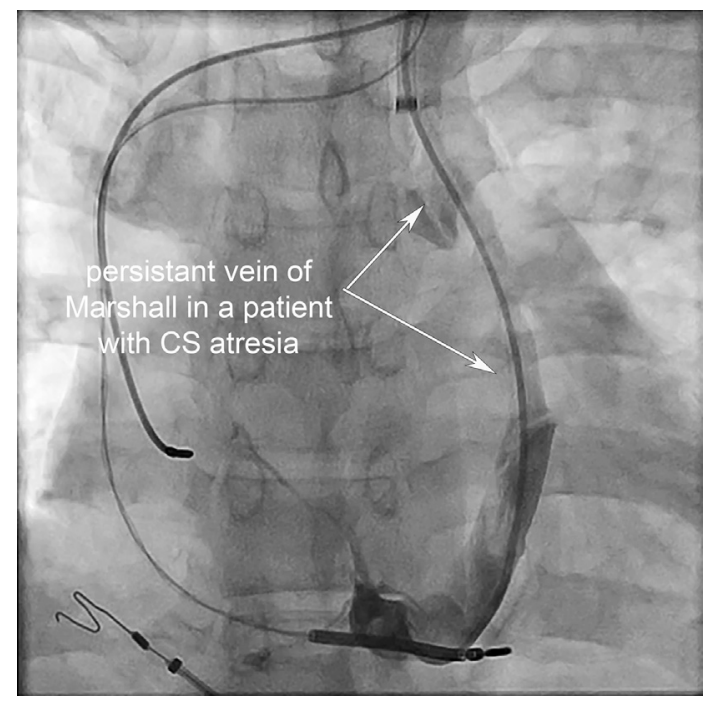

Fig. 5. Patient with CS atresia and persistent vein of Marshall through which the LV lead can be implanted. Watch Video 2 for details (https://www.youtube.com/ watch?v=1 mLRpwJ1k8A). (Courtesy of Seth J. Worley, MD, Washington, DC.)

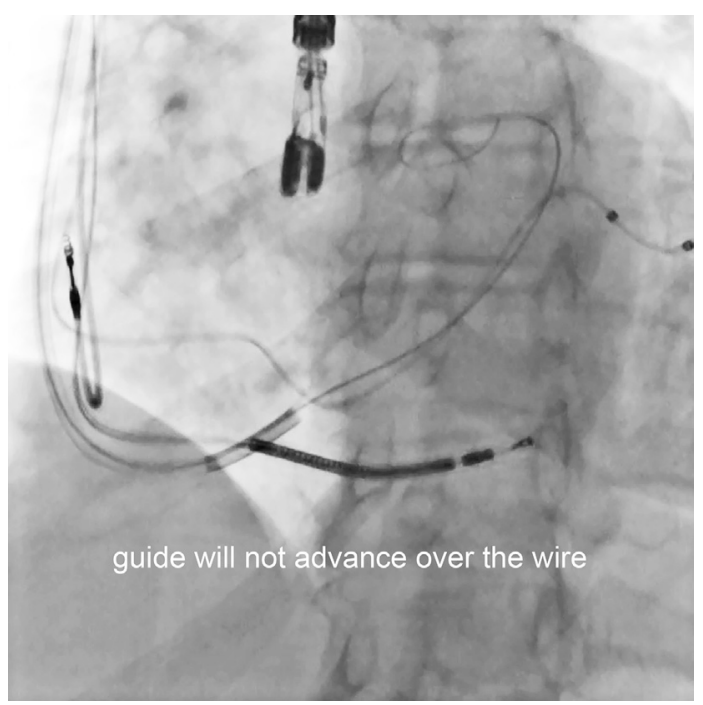

Fig. 6. A patient with a stenotic CS where the device company delivery systems could not be advanced. Using the Merit "Vertebral Vein Selector" and Cook Amplatz wire, CS cannulation was successful. The vein selector/Amplatz wire technique also works well for a tortuous CS. Watch Video 3 for details (https:// www.youtube.com/watch?v=SB4nNBmy-3g). (Courtesy of Seth J. Worley, MD, Washington, DC.)

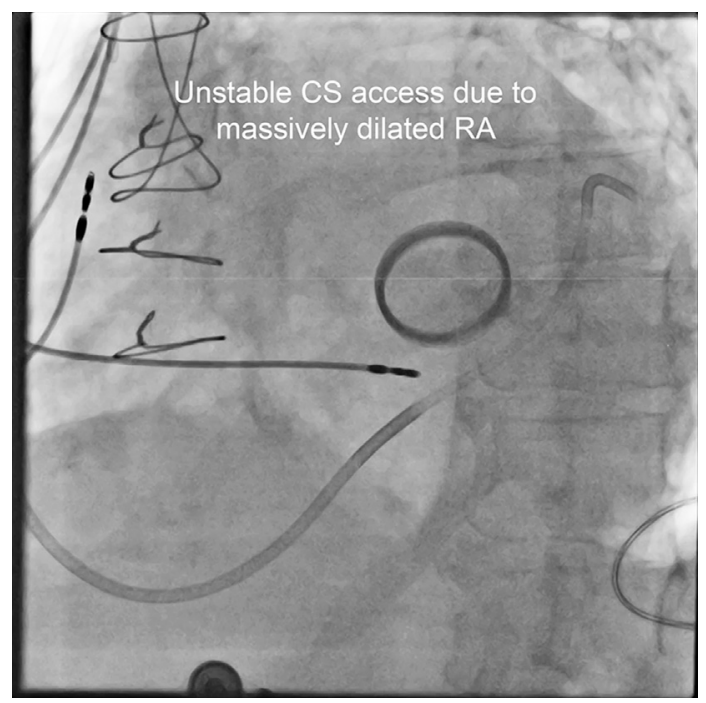

Fig. 7. Using several different device company delivery systems, it was impossible to establish stable CS access in this patient with a massively dilated RA. The Jumbo Worley sheath and Cook Amplatz support provide stable CS access followed by successful LV lead delivery. Sometimes switching from the Standard Worley sheath to the Jumbo Worley sheath facilitates initial CS cannulation. Watch Video 4 for details (https://www.youtube.com/watch?v=5P7gJltePP8).

(Courtesy of Seth J. Worley, MD, Washington, DC.) 


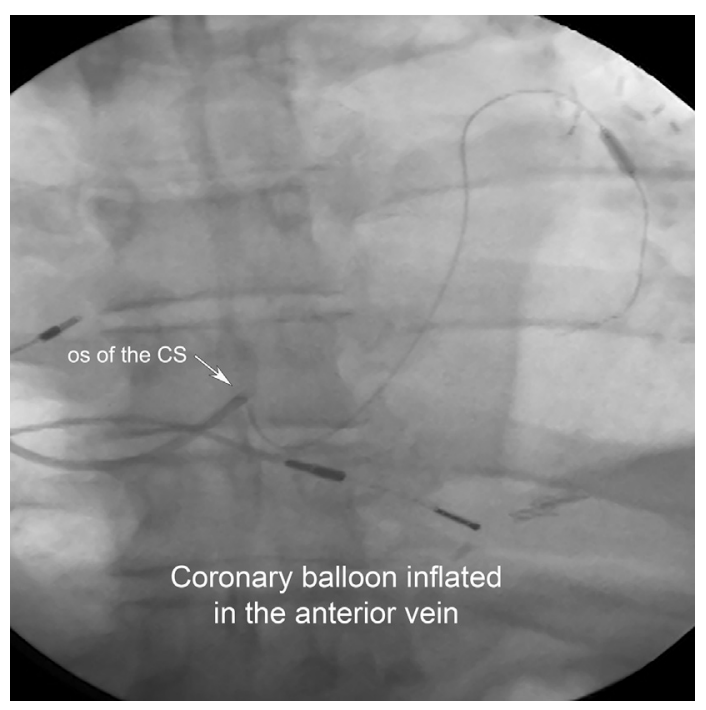

Fig. 8. Previous attempts to cannulate the "drain pipe" were unsuccessful. As the catheter was advanced, the tip served as a fulcrum and the proximal segment was dropped into the RA. The CS was successfully cannulated using anchor balloon technique. Watch Video 5 for details (https://www. youtube.com/watch?v=hmXtcsVjsyw). (Courtesy of Seth J. Worley, MD, Washington, DC.)

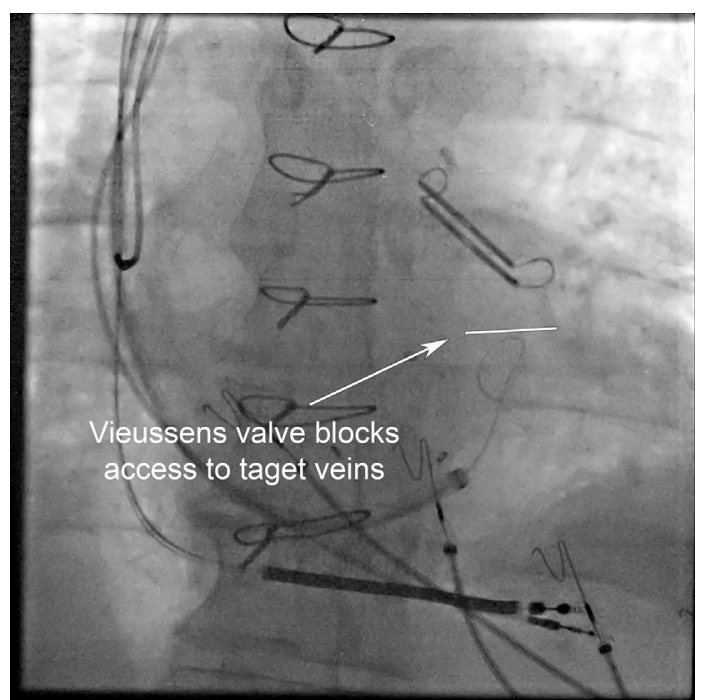

Fig. 9. Vieussens valve blocked access to target veins resulting in 2 failed attempts at LV lead implantation. Using the "Vertebral Vein Selector" and contrast injection system, the valve was crossed, and lead was placed. Watch Video 6 for details (https://wwww. youtube.com/watch?v=SdTEB3R6W8M). (Courtesy of Seth J. Worley, MD, Washington, DC.)

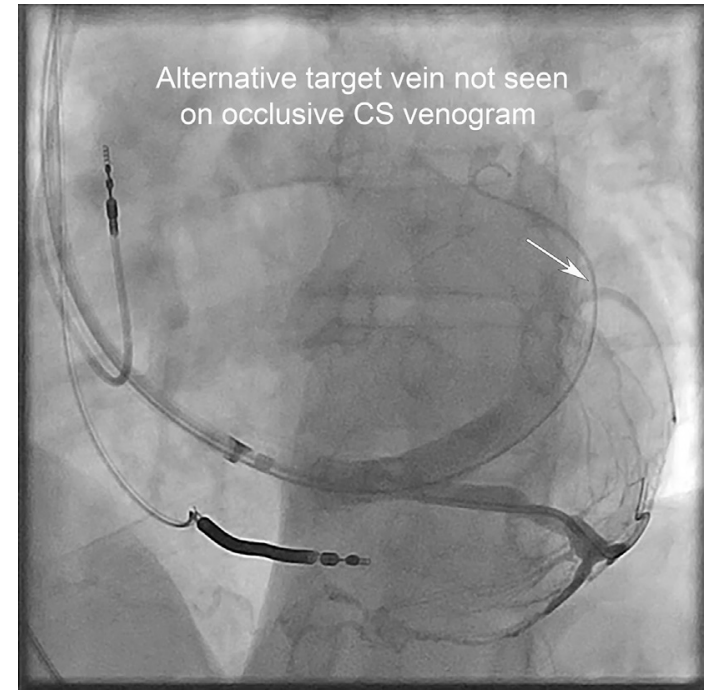

Fig. 10. Selective venogram using the Vein Selector reveals a CS target vein not seen on occlusive venography. Watch Video 7 for details (https://www. youtube. $\mathrm{com} /$ watch? $\mathrm{v}=23 \mathrm{nhCd} 2 \mathrm{~h} 9 \mathrm{Gw}$ ). (Courtesy of Seth J. Worley, MD, Washington, DC.)

watch Video 10 (https://www.youtube.com/ watch?v=19gEdLSNcFg).

Problem $=$ angulated stenotic target vein near the ostium of the CS (Fig. 14); solution = the "Hook Vein Selector" telescoped in the renal LVI subselector through the 9-French sheath supported by the Cook Amplatz wire. For details on

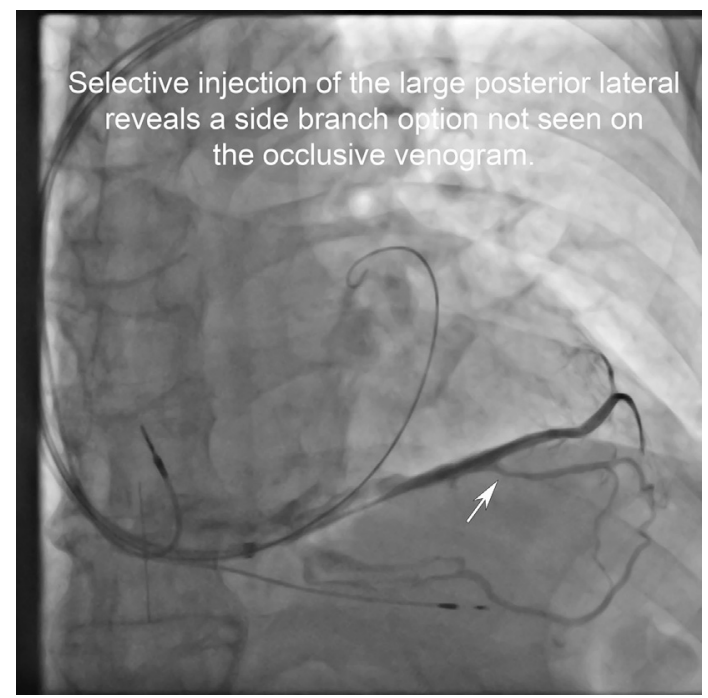

Fig. 11. Selective venogram using the Vein Selector reveals a side branch off the target vein not seen on occlusive venography. Watch Video 8 for details (https://www.youtube.com/watch?v=mWVeJIYC92s). (Courtesy of Seth J. Worley, MD, Washington, DC.) 


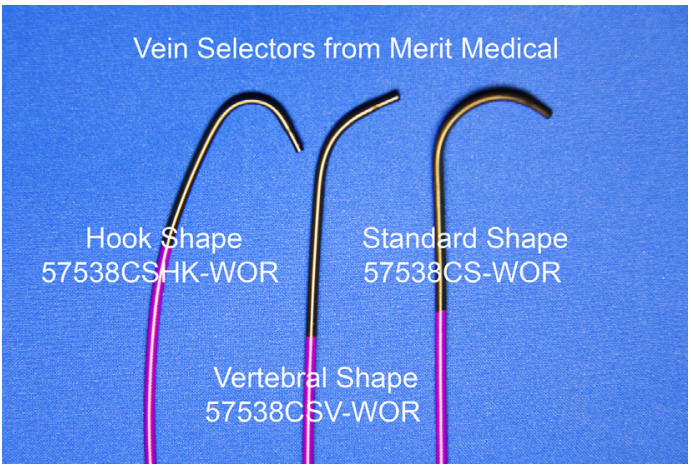

Fig. 12. There are 3 vein selector shapes designed to for specific anatomy. Watch Video 9 for details (https://www.youtube.com/watch?v=IsawLqHGq-g). (Courtesy of Seth Worley, MD, Washington, DC; and Merit Medical Systems, Inc, South Jordan, UT.)

how to use the Hook Vein Selector and Amplatz support wire technique for target veins near the CS os, watch Video 11 (https://www.youtube. $\mathrm{com} /$ watch? $\mathrm{v}=\mathrm{fQynQNB}-\mathrm{jp} 0$ ).

Problem $=$ target vein at the ostium of the CS (Fig. 15); solution = the "Vertebral Vein Selector" telescoped in the renal LVI subselector through the 9-French sheath supported by the Cook Amplatz wire. For details on how to use the Amplatz support wire technique for target veins at or near the CS, watch Video 12 (https://www. youtube.com/watch? $v=4 X Y$ wfi5Ba6U).

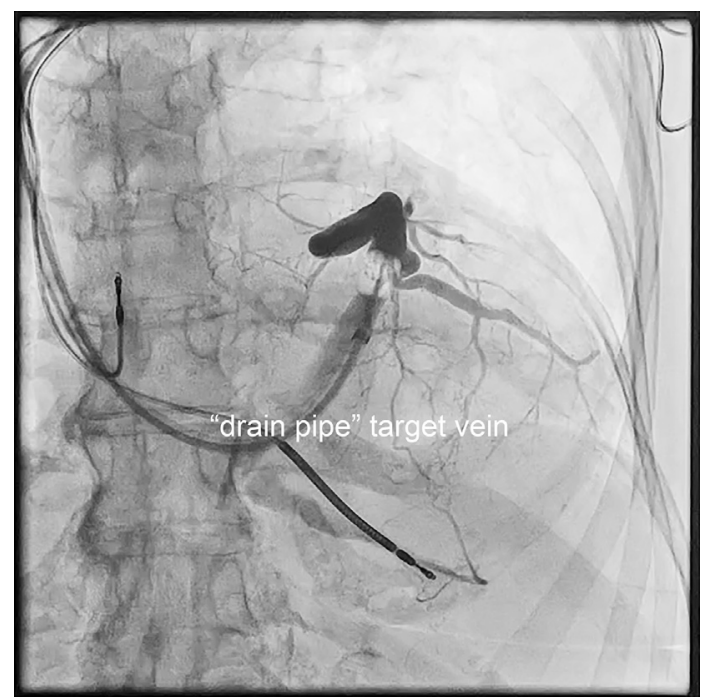

Fig. 13. Target veins with "drain pipe" origin from the CS can be difficult to implant. This patient had a previously failed attempt. The "Hook Vein Selector" telescoped inside the "Renal LVI" subselector makes implantation possible. Watch Video 10 for details (https://www.youtube.com/watch?v=19gEdLSNcFg). (Courtesy of Seth J. Worley, MD, Washington, DC.)

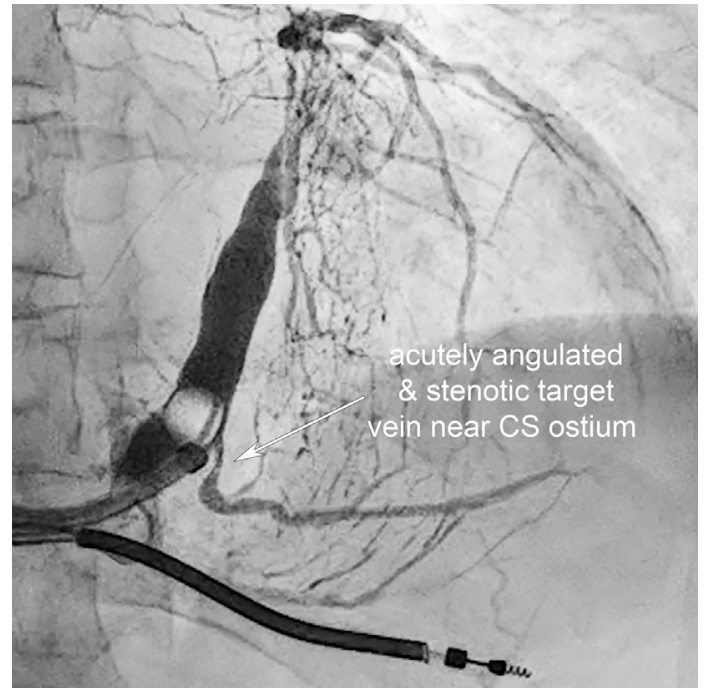

Fig. 14. Target veins with acute angulation near the ostium of the CS can be implanted using the "Hook Vein Selector" telescoped inside the "Renal LVI" subselector with the Worley sheath supported by the Cook Amplatz wire. For details, watch Video 11 (https://www.youtube.com/watch?v=fQynQNB-jp0). (Courtesy of Seth Worley, MD, Washington, DC.)

Problem $=$ angulated target vein when a subselector cannot be advanced for LV lead delivery (Fig. 16); solution = the "Standard Vein Selector" telescoped in the renal LVI subselector

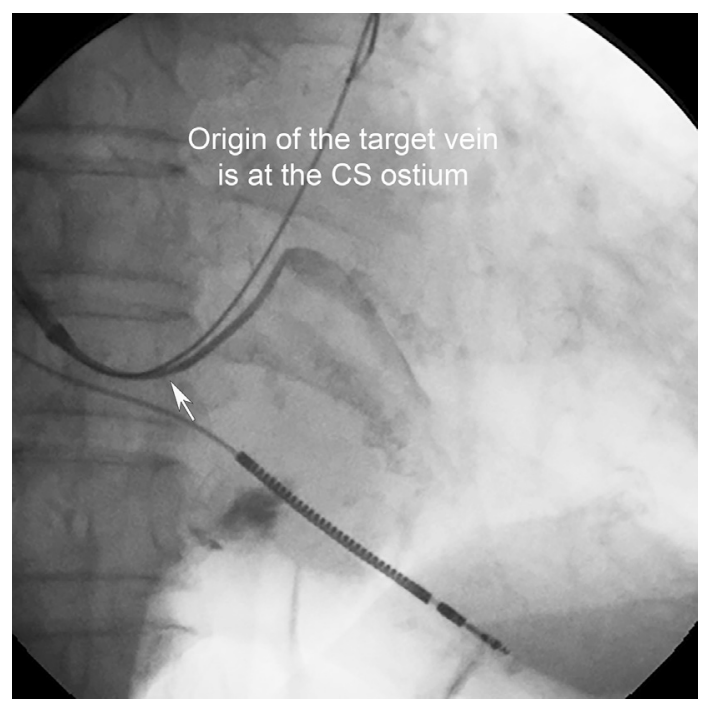

Fig. 15. Target vein with its origin at the CS ostium can be implanted easily using the Cook Amplatz support wire technique and "Vertebral Vein Selector" telescoped inside the "Renal LVI" subselector. Watch Video 12 for details (https://wwww.youtube.com/ watch?v=4XYwfi5Ba6U). (Courtesy of Seth Worley, MD, Washington, DC.) 


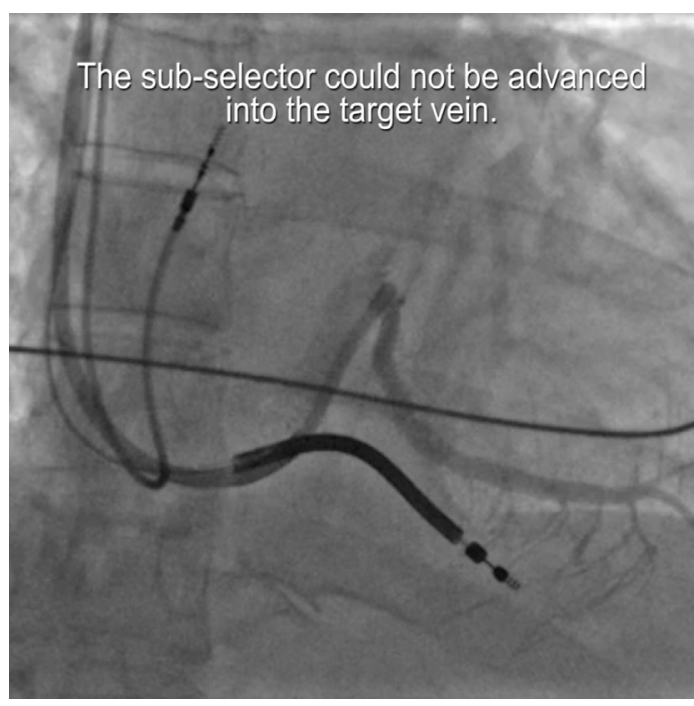

Fig. 16. LV lead could not be placed in this angulated target vein using device company subselector despite multiple attempts by several physicians over the course of several hours. Using the "Standard Vein Selector" telescoped inside the "Renal LVI" subselector, the LV lead was easily placed in 15 minutes. See Video 13 for details (https://www.youtube.com/ watch? $v=0$ apbC0kPumo\&t=7s). (Courtesy of Seth Worley, MD, Washington, DC.)

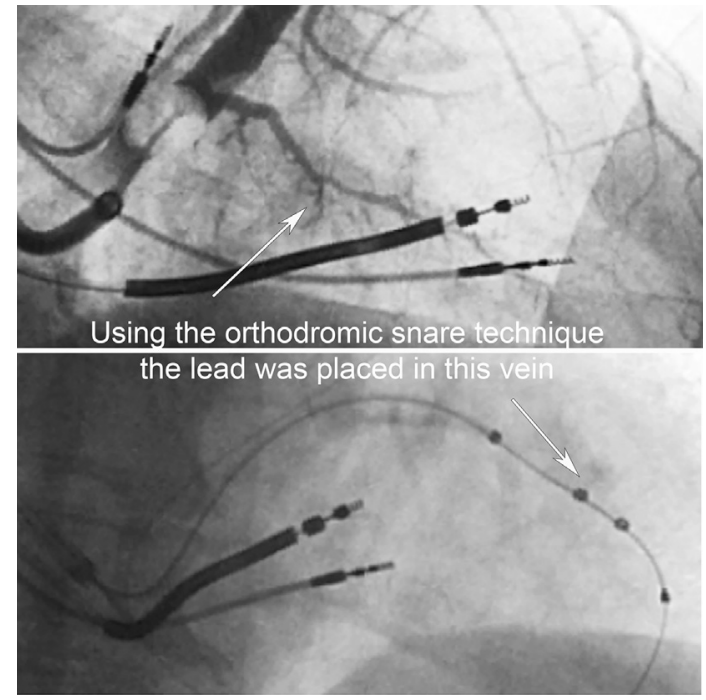

Fig. 17. Target vein too small for LV lead placement using traditional techniques successfully implanted via the orthodromic snare technique. Watch Video 14 for details on how to implement the orthodromic snare technique (https://www.youtube.com/watch? $\mathrm{v}=\mathrm{tmr} 8 \mathrm{z} 7 \mathrm{bltN0}$ ). (Courtesy of Seth Worley, MD, Washington, DC.)

\section{Box 4 \\ Sheaths, catheters, and wires required for the orthodromic and antidromic snare technique}

1. Worley Advanced Standard Curve; Order no. WOR-CSG-B1-09 Merit Medical or Worley Advanced Jumbo Curve; Order no. WOR-CSG-B2-09. Merit Medical.

2. Worley Standard Vein Selector $(5$ French $\times 75 \mathrm{~cm}$ ) Merit Order no. 57538CS-WOR Merit Medical.

3. Worley Vert Vein Selector $(5$ French $\times 75 \mathrm{~cm}$ ) Merit Order no. 57538CSV-WOR Merit Medical.

4. Worley Hook Vein Selector $(5$ French $\times 75 \mathrm{~cm}$ ) Merit Order no. 57538CSHK-WOR Merit Medical.

5. Contrast Injection System Worley Advanced Kit 1 CAK 1 (comes with contrast bowl and labels) (order no.K12-WORLEY1 Merit Medical).

6. 4-French Impress KA 2 Hydrophilic Angiographic Catheter 4-French 65-cm (Order no. 46538KA2-H Merit Medical).

7. 0.014-in CholCE PT (Polymer Tip) Straight Tip Light Support 180-cm (order no. 1211-01 Boston Scientific).

8. 0.014-in CholCE PT (Polymer Tip) Straight (not angled) Tip Light Support 300-cm Boston Scientific.

9. Cook Amplatz Extra Stiff Wire Guide, 0.035 in, 180-cm, 3-mm-tip curve Cook (THSCF-35-180-3-AES) (another ref. no. on the package is G03565) (do not substitute).

10. Snare (Micro) 10-mm loop/4-French snare catheter Merit One Snare ONE 1000 (snare = 10-mm loop, $120-\mathrm{cm}$ length; catheter $=4$-French $100-\mathrm{cm}$ length inside diameter $1.02 \mathrm{~mm}$ ). This $10-\mathrm{mm}$ snare fits into the 9-French internal diameter CSG Worley beside the vein selector (Order no. ONE100 Merit Medical).

11. Microcatheter in case the snared wire gets bent and for the antidromic snare technique Merit SureCross Support Catheter (length $90 \mathrm{~cm}$, wire diameter 0.014 in, tip diameter 0.020 in [1.52 French]) Catalog no. SC1490. 
Box 5

Orthodromic snare technique step by step

Engage the target vein with the appropriate shape "Vein Selector."

Advance a straight light support polymer tip wire into the vein (Choice PT Floppy Boston Scientific).

Once the wire is in the vein, advance the vein selector 3 to $7 \mathrm{~mm}$ over the wire into the vein.

If the wire becomes bent as it is advanced, it may not traverse the collaterals. Once it is bent, withdrawing the wire back into the vein selector does not straighten the wire. Do not remove the bent wire. Add a second wire. The bent wire orients and supports the vein selector, so the second wire remains straight as it exits into the vein.

The straight second wire is advanced through the collaterals into an adjacent vein (exit vein) and back into the CS.

If there is difficulty advancing the wire through the collaterals, one option is to add a microcatheter as follows: (1) remove the Y adapter from the hub of the injection system; (2) advance a 0.014-in microcatheter (SureCross) over the existing wire; (3) exchange the existing wire for a fresh wire; (4) the microcatheter provides support to advance the fresh wire through the collaterals.

To confirm that the wire is back in the CS, check the left anterior oblique (LAO) and RAO projections. Once the wire traverses the collaterals, load the 10-mm loop into the 4-French snare catheter.

Holding the vein selector in position, advance the 4-French 10-mm snare into the 9-French sheath beside the 5-French "Vein Selector." (As the snare is advanced, hold the vein selector [or lead] in place; otherwise friction can cause the vein selector [or lead] to advance.)

How the wire is snared depends on the direction the wire takes once it reaches the CS: either (1) up the CS toward the great cardiac vein; or (2) out of the CS into the RA.

1. When the wire turns up into the CS toward the great cardiac vein, the snare is positioned in the body of the CS above the "exit vein" (usually the middle cardiac vein (MCV)) and the wire is advanced into the open loop.

2. When the wire exits into the RA, the vein selector is held in place and the snare is positioned over the ostium of the "exit vein" (usually the MCV). It is critical to check the RAO projection to confirm that the snare is positioned over the MCV. The loop can appear to be properly positioned in the anterior, posterior and LAO projection, but when advanced, the wire is not captured by the snare.

After $15 \mathrm{~cm}$ of wire is through the loop, the snare is closed on the wire. It is important to tighten the snare on the stiff part of the wire to avoid bending the wire or pulling off the tip.

To secure the snared wire: (1) the right hand presses the open hemostat against the hub of the snare catheter; (2) the left hand pulls the snare loop tight against the wire; (3) the hemostat is closed on the snare at the hub of the snare catheter.

With wire snared, the sheath is withdrawn into the RA (holding the snare and vein selector in place).

To prevent the snare from being pulled down into the sheath when tension is placed on the wire, a second snap is placed on the snare catheter where it enters the hub of the sheath.

To advance the lead, a rail is created by placing tension on the snared wire.

Tension on the wire creates a rail over which the lead could be advanced despite the stenosis and tortuosity. In some cases, even with the snare, the lead will not advance without venoplasty.

With the wire still snared and the lead in place, thresholds are tested. Thresholds may change slightly once the snare is released and the wire is removed. The presence or absence of phrenic pacing does not change.

Once satisfactory thresholds are achieved, the snare system is removed.

To prevent any chance of lead dislodgment, the sheath is removed with the wire still snared. Again, thresholds may change only slightly once the snare is released and the wire is removed.

To open the snare, the loop is advanced 3 to $5 \mathrm{~mm}$ into the snare catheter.

Withdraw the wire through the open snare until the tip is clear of the snare but still in the pacing lead. With the wire free of the snare, the loop is withdrawn into the snare catheter. Hold the lead in place and remove the snare.

After a final adjustment of slack in the LAO projection, the wire is withdrawn. 
Special Situations

Inability to Capture the Wire with the Snare

First, confirm the position of the loop relative to the exit vein in the RAO projection. In some cases, it can be very difficult to snare the wire as it exits the MCV into the RA. One option is to position the snare inside the MCV. To get the snare in the MCV, start by loading a glide wire into the snare catheter. Advance the glide wire/snare catheter into the sheath beside the vein selector. Advance the glide wire into the MCV. Advance the snare catheter over the glide wire into the MCV. Remove the glide wire and load the snare loop into the snare catheter. Deploy the loop in the MCV and advance the wire into the open snare. Alternatively, the snare catheter can be replaced with a 4-French angled catheter (KA2) to aim the loop of the snare in the desired direction.

\section{Wire Becomes Bent by the Snare}

On occasion, the snare bends the wire usually when the snared wire is inadvertently pulled back into the sheath. To prevent bending the wire, withdraw the sheath into the RA once the wire is snared. When the wire is bent, it can be impossible to remove from the pacing lead or the bent tip can fracture and embolize. When the wire is bent, follow these steps: (1) keep the wire snared; (2) remove the pacing lead; (3) flush and wipe the wire; (4) advance the microcatheter over the wire through the collaterals up the snare; (4) attach a Y adapter to the hub of the microcatheter, tighten the hemostatic valve, and flush the microcatheter with hepatized saline using a 1- to $3-\mathrm{mL}$ syringe; (5) loosen the hemostatic valve and advance the wire into the microcatheter while the wire is withdrawn into the sheath with the snare; (6) once the bent wire is removed, flush the microcatheter and insert a fresh wire; (7) reinsert the snare into the CS; (8) keeping the wire in place, withdraw the microcatheter and resnare the wire; (8) replace the microcatheter with the pacing lead.

makes it possible to easily advance the subselector into the target vein for LV lead delivery. For details on how the wire-stabilized vein selector is used to augment a subselector, watch Video 13 (https://www.youtube.com/watch?

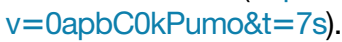

Problem $=$ tortuous small and or stenotic target vein (Fig. 17); solution = orthodromic snare technique (Boxes 4 and 5). ${ }^{7}$ Also watch Video 14 for details on how to implement the orthodromic snare technique (https://www.youtube. com/watch?v=tmr8z7bltN0).

Problem $=$ inability to advance a wire into the target vein (Fig. 18); solution = antidromic snare technique (Boxes 4 and 6, Video 15). Watch Video 15 for details on how to implement the antidromic
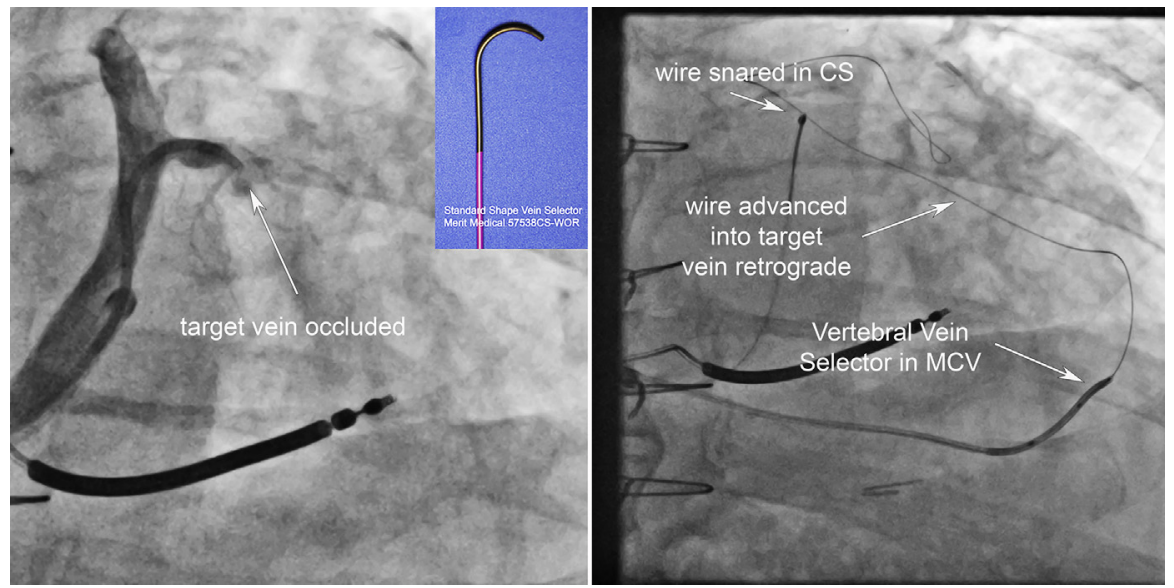

Fig. 18. Unable to advance a wire into the occluded target vein occluded from prior unsuccessful LV lead placement. Wire advanced out of the target vein using collaterals from the middle cardiac vein. Lead placed using the antidromic snare technique and coronary vein venoplasty. Watch Video 15 for details on how to implement the antidromic snare technique (https://www.youtube.com/watch?v=ez4Kvd2iYiE) and (see Box 6) for a detailed step by step discussion of the antidromic snare technique. (Courtesy of Seth Worley, MD, Washington, DC; and Merit Medical Systems, Inc, South Jordan, UT.) 
1. The first step is to engage a branch with collaterals to the target vein, usually the MCV. The 9-French internal diameter "Worley" sheath (WOR-CSG-B1-09 Merit Medical) provides the option to use the support wire technique to easily engage the MCV. The support wire technique is not an option with a 7-French CS access catheter. See the Amplatz support wire technique for details.

2. The shape of the "Vertebral Vein Selector" is well suited for cannulation of the MCV and target veins below the Vieussens valve. Full-strength contrast is injected to define the collaterals to the target vein. A puff of contrast from the injection system confirms the tip of "Vein Selector" has dropped into the MCV. An angled 0.035-in glide wire is advanced into the MCV.

3. With the Worley sheath stabilized by the Cook Amplatz wire, the "Vein Selector" is advanced into the MCV over a glide wire. The glide wire is removed and a puff of contrast is injected to define the collaterals from the MCV to the target vein. In most cases the angle of the "Vertebral Vein Selector" works well with the angle of the collaterals.

4. A $300-\mathrm{cm}$ light support (floppy) straight polymer jacketed angioplasty wire is advanced into the collaterals (Choice PT Floppy).

5. The position of the wire is assessed with a puff of contrast through the vein selector. The $300-\mathrm{cm}$ wire is advanced through the collateral leading to the target vein and then back into the CS.

6. If there is any difficulty advancing the wire through the collaterals, the $Y$ adapter on the vein selector is removed and a SureCross microcatheter is advanced over the wire through the vein selector into the collateral. Because the "Vein Selector" is $75 \mathrm{~cm}$ and the SureCross is $90 \mathrm{~cm}$, it is important to remove the $Y$ adapter to load the catheter over the wire.

7. Once the wire is back in the CS, advance the snare catheter over the $180-\mathrm{cm}$ Cook Amplatz to support the wire into the CS. Once the snare catheter is in the mid-CS, the Amplatz wire is removed.

8. With the tip of the 4-French snare catheter in the CS, load the $10-\mathrm{mm}$ loop using the introducer. The $10-\mathrm{mm}$ loop is advanced through the snare catheter until it is deployed in the mid-CS.

9. The angioplasty wire is withdrawn toward the target vein until the tip is beyond the loop of the snare. The wire is then advanced into the open snare. The loop of the snare is closed on the wire by advancing the snare catheter.

10. Once the tip of the wire is secured by the snare, the goal is to use the snare to withdraw $120 \mathrm{~cm}$ of the angioplasty wire into the sheath and out into the pocket. However, there is the potential for the cheese cutter effect.

11. To prevent the cheese cutter effect, the wire is covered with a microcatheter. The wire is advanced through the microcatheter, not pulled through the collaterals.

12. The $Y$ adapter is removed, and the SureCross is advanced over the wire, through the vein selector into the collaterals.

Again, because the "Vein Selector" is $75 \mathrm{~cm}$ and the SureCross is $90 \mathrm{~cm}$, it is important to remove the $Y$ adapter to load the catheter over the wire.

Hydrophilic microcatheter 90-cm, 0.14-in Iumen 1.5-French tip (SureCross Support Catheter Merit Medical).

Alternatively, the vein selector is removed before the microcatheter is advanced through the collaterals.

With the wire snared in the CS, the microcatheter is advanced through the collaterals.

CAUTION: Failure to flush the microcatheter can result in seizure of the wire.

Do not use the snare to pull the wire through the microcatheter.

The distal end of the wire is advanced into the microcatheter while the tip of the lead is withdrawn into the 9-French Worley sheath with the snare.

The wire should advance easily into the microcatheter to provide slack for the snare to withdraw into the sheath.

The process of advancing the proximal end of the wire into the microcatheter and withdrawing the snared end is continued until $80 \mathrm{~cm}$ of wire is externalized into the pocket.

The wire should advance easily into the microcatheter to provide slack for the snare to withdraw into the sheath.

If there is any difficulty advancing the wire into the microcatheter's STOP, close the hemostatic valve and flush the microcatheter with a 1- to 2-mL syringe.

The snare is opened, and the bent wire is removed with a pair of scissors. 
The cut snared end (tip) of the wire is back-loaded into the tip of the pacing lead.

The proximal end of the wire is secured at the hub of the $Y$ adapter used to flush the microcatheter.

With tension on the distal end of the wire, the pacing lead is advanced into the target vein.

Before advancing the pacing lead, be certain the sheath is out of the CS. The sheath can get jammed into the crux between the MCV (wire exit vein and the CS) preventing the lead from advancing.

In some cases, the lead may not advance because there is too much tension on the wire.

Temporarily relaxing tension while advancing can help the lead to advance, particularly around tight curves.

Another situation where there can be difficulty advancing the LV lead is when you forget to withdraw the tip of the microcatheter back into the microcatheter.

To allow the lead to advance, the microcatheter must be withdrawn into the collaterals.

Once the lead is in place and thresholds confirmed, the sheath is peeled away. The wire is removed through the microcatheter.

If there is any difficulty moving the wire, close the hemostatic valve and flush the microcatheter.

Also flush the leads as described below.

Special Cases

1. In some patients, the "Vertebral Vein Selector" is not the correct shape to engage the collateral to the target vein. When this occurs, switch to a "standard" or "hook" vein selector.

2. Although the collaterals leading to the target vein are often found in the MCV, they may be found connecting to other adjacent veins.

3. If the microcatheter will not advance through the collaterals (rare with the 0.014-in SureCross), the microcatheter can be exchanged for an over-the-wire 1.25 - $\mathrm{mm}$ coronary balloon. Once the balloon is through the collaterals into the CS, the lumen of the balloon is used as the conduit through which to advance the wire.

4. Usually the wire is snared in the CS before the microcatheter is advanced. However, sometimes the wire traverses the collaterals to the target vein but will not advance from the target vein into the CS. To get the wire to exit the target vein, the $Y$ adapter is removed and the 0.014 -in microcatheter is advanced over the wire through the collaterals into the target vein. The support of the microcatheter facilitates wire passage out of the target vein into the CS. Once the microcatheter is in the target vein, a bent wire can be replaced without the risk of not being able to cross the collaterals.

5. The "Vein Selector" can be replaced with the 0.014-in microcatheter and advanced through the collaterals into the target vein to facilitate wire passage out of the target vein into the CS. The microcatheter provides support to advance the wire and/or the bent wire can be replaced with a new wire. The wire can then be advanced into the open snare and secured.

snare technique (https://www.youtube.com/ watch?v=ez4Kvd2iYiE).

\section{REFERENCES}

1. [Chapter 30] Worley SJ. Coronary Sinus Lead Implantation. In: Ellenbogen KA, Wilkoff BL, Kay GN, et al, editors. Coronary sinus lead implantation in: clinical cardiac pacing, defibrillation and resynchronization therapy. 45th edition. Philadelphia (PA): Elsevier; 2017.

2. Worley SJ, Gohn DC, Pulliam RW, et al. Subclavian venoplasty by the implanting physicians in 373 patients over 11 years. Heart Rhythm 2011;8(4):526-33.

3. [Chapter 13] Worley SJ. How to perform venoplasty for access. In: Worley SJ, editor. How-to manual for pacemaker and ICD devices: procedures and programming. Hoboken (NJ): John Wiley \& Sons. Inc; 2017. p. 97-109.

4. Marcial JM, Worley SJ. Venous system interventions for device implantation. Card Electrophysiol Clin 2018;10(1):163-77.

5. Jackson KP, Hegland DD, Frazier-Mills C, et al. Impact of using a telescoping-support catheter system for left ventricular lead placement on implant success and procedure time of cardiac resynchronization therapy. Pacing Clin Electrophysiol 2013;36(5):553-8.

6. Worley SJ. How to use balloons as anchors to facilitate cannulation of the coronary sinus left ventricular lead placement and to regain lost coronary sinus or target vein access. Heart Rhythm 2009;6(8):1242-6.

7. Worley SJ, Gohn DC, Pulliam RW. Goose neck snare for LV lead placement in difficult venous anatomy. Pacing Clin Electrophysiol 2009;32:1577-81. 